\title{
Epidemic spreading in evolving networks
}

\author{
Yonathan Schwarzkopf, ${ }^{1,2,3}$ Attila Rákos, ${ }^{1,4}$ and David Mukamel ${ }^{1}$ \\ ${ }^{1}$ Department of Physics of Complex Systems, Weizmann Institute of Science, Rehovot 76100, Israel \\ ${ }^{2}$ California Institute of Technology, Pasadena, California 91125, USA \\ ${ }^{3}$ Santa Fe Institute, Santa Fe, New Mexico 87501, USA \\ ${ }^{4}$ Research Group for Condensed Matter Physics of the Hungarian Academy of Sciences, \\ Budapest University of Technology and Economics, 1111 Budapest, Hungary
}

(Received 22 January 2010; revised manuscript received 24 August 2010; published 24 September 2010)

\begin{abstract}
A model for epidemic spreading on rewiring networks is introduced and analyzed for the case of scale free steady state networks. It is found that contrary to what one would have naively expected, the rewiring process typically tends to suppress epidemic spreading. In particular it is found, that as in static networks under a mean-field approximation, rewiring networks with degree distribution exponent $\gamma>3$ exhibit a threshold in the infection rate below which epidemics die out in the steady state. However the threshold is higher in the rewiring case. For $2<\gamma \leq 3$ no such threshold exists, but for small infection rate the steady state density of infected nodes (prevalence) is smaller for rewiring networks.
\end{abstract}

DOI: 10.1103/PhysRevE.82.036112

PACS number(s): 89.75.Fb, 89.90.+n, 05.40.-a

\section{INTRODUCTION}

Epidemic spreading can be thought of as occurring on complex networks where the nodes of the network represent individuals and the links represent various interactions among those individuals. For example the spreading of diseases can be thought of as occurring over the network of human contacts [1] and the spreading of computer viruses as occurring over the internet [2,3]. Models of epidemic spreading over networks have been studied extensively in recent years (for reviews see [4,5]). Typically, the underlying network in these models is considered to be static while the state of the individuals residing on its nodes can change from infected to noninfected according to some dynamical rules. One is then interested in studying the evolution of an infected region in time, the average density of infected nodes in steady state (prevalence) and the way they are affected by the statistical properties of the network and the infection rates.

In general, networks can be characterized by the connectivity of their nodes. The connectivity (degree) $k$ of a node is defined as the number of links connected to the node. The degree distribution of a network $\mathcal{P}(k)$ is defined as the probability of a randomly chosen node to have a degree $k$. Many networks such as social networks, the internet and the World Wide Web (WWW) have been found to be scale free (SF) [6-10], meaning that the degree distribution follows a power law

$$
\mathcal{P}(k) \sim k^{-\gamma} .
$$

In the thermodynamic limit one can divide SF networks into two classes based on the exponent $\gamma$. For $\gamma>3$ the second moment of the degree distribution is finite and as such the system exhibits finite degree fluctuations. For $2<\gamma \leq 3$ the second moment diverges resulting in infinitely large degree fluctuations. In the present study we only consider networks with a finite degree distribution corresponding to $\gamma>2$. Interestingly, many real networks have been measured to belong to the second class having $2<\gamma \leq 3$ [10].

Studies of models of epidemic spreading over static networks have shown that in networks with a degree distribu- tion that is not too heavy tailed, for which all the moments exist, the prevalence, $\rho$ vanishes for sufficiently small infection rates $\lambda$. The prevalence becomes nonzero only beyond a threshold rate $\lambda_{c}$. On the other hand, it has been recently shown that for power-law networks, for which the $m>\gamma-1$ moment does not exist, the prevalence is nonzero for any infection rate, and no threshold exists [11]. Thus, epidemics are much harder to stop in static networks with power-law degree distributions. The result in [11] contradicts the meanfield (MF) results [12-15] that state that for power-law degree distributions with a finite second moment, i.e., $\gamma>3$, a threshold exists while for networks with $2<\gamma \leq 3$, for which the second moment of the degree distribution diverges, no threshold exists.

In many cases networks are not static but rather evolve in time, for example via rewiring processes. By rewiring we refer to a process in which edges are detached and reattached (shuffled) according to some dynamic rule. Steady states of rewiring networks have been studied in the past. It has been shown that depending on the average degree and the rewiring rates, networks may reach an SF steady state, with an exponent $\gamma$ which can be expressed in terms of the dynamical rates $[16-18]$.

In the present paper, we consider the MF description of epidemic spreading over rewiring networks. On such networks, the disease can spread at a given time through the links which are present at that time. We find that as in the static case the MF approximation suggests a nonvanishing threshold value of the infection rate, $\lambda_{c}$, exists for $\gamma>3$. Below this threshold the prevalence (fraction of infected individuals) vanishes while above it the prevalence is nonzero. For $2<\gamma \leq 3$, in the MF approximation, no such threshold exists and the steady state prevalence is nonzero for any $\lambda$ $>0$. However, contrary to what one would have naively expected, epidemic spreading in our model is not necessarily enhanced by the dynamics of the network. For $\gamma>3$ the threshold $\lambda_{c}$ is found to be larger than that of the corresponding static network. Also, for $2<\gamma \leq 3$ the prevalence at small $\lambda$ is found to be smaller than that of the corresponding static network. 
The paper is organized as follows: in Sec. II, we review known results on epidemic spreading in static networks and on networks with rewiring dynamics. In Sec. III, we study epidemic spreading on evolving networks using mean-field calculations and numerical simulations. Our results are summarized in Sec. IV.

\section{REVIEW OF KNOWN RESULTS}

\section{A. Epidemic spreading in static networks}

A number of models of disease spreading have been introduced and studied in the past. In the present work we use the susceptible infected susceptible (SIS) model $[4,5,10,12,13,16,19]$. In this model a healthy individual, with respect to the disease, may be infected through interaction with diseased individuals. Meaning, that a susceptible node may be infected through a link connecting it to an infected node, which we will refer to as his neighbor. Once an individual is infected he may become susceptible again by being spontaneously cured from the disease. The curing process does not immune the individual and it can be reinfected.

The continuous time dynamics of an epidemic in the SIS model is defined by two stochastic processes using two parameters:

(i) $\lambda$-Infection rate;

(ii) $\delta$-Rate of recovery.

An infected node is spontaneously cured with a rate $\delta$ which we choose to be equal to 1 by adjusting the time scale. On the other hand a susceptible node gets infected with rate $\lambda$ from each of its infected neighbors. Thus, the rate a node is infected depends linearly on the number of infected neighbors. This model of infection is different from the model explored in $[12,13,16,19]$ where the infection rate is independent of the number of infected neighbors. However, both models behave similarly near the threshold for an endemic state and we expect our conclusions to hold for both models.

The problem is addressed using a mean-field (MF) approach and numerical simulations. The MF approach neglects correlation in infection between nodes in the sense that for any pair of nodes $i, j$ we have $\left\langle\eta_{i} \eta_{j}\right\rangle=\left\langle\eta_{i}\right\rangle\left\langle\eta_{j}\right\rangle$ where $\eta$ $=0,1$ is a parameter indicating whether a node is susceptible or infected, respectively. As an order parameter we use the prevalence of the disease, the density of infected nodes in the network, defined as $\rho \equiv N_{\text {infected }} / N$. Hence, our problem is reduced to a contact equation for the order parameter $\rho$. Since we are interested in formulating the problem for any degree distribution, as was previously done in $[12,13]$, we shall distinguish between nodes of different degree by defining $\rho_{k}$ as the fraction of diseased nodes of degree $k$. The total prevalence is thus given by

$$
\rho=\sum_{k=0}^{\infty} \rho_{k} \mathcal{P}(k) .
$$

The MF contact equation has the following form:

$$
\frac{\partial \rho_{k}}{\partial t}=-\rho_{k}+\lambda k \ell\left(1-\rho_{k}\right) .
$$

With $\ell$ being the density of "infected links" defined as

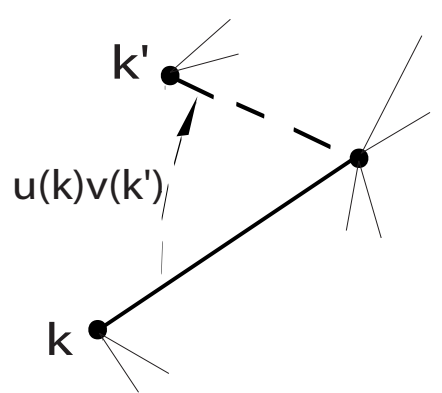

FIG. 1. Rewiring of a link from a node with degree $k$ to a node with degree $k^{\prime}$ with a rate $u(k) v\left(k^{\prime}\right)$.

$$
\ell=\sum_{k=0}^{\infty} \frac{k \mathcal{P}(k)}{\langle k\rangle} \rho_{k} .
$$

Note that

$$
\mathcal{P}^{*}(k)=\frac{k \mathcal{P}(k)}{\langle k\rangle}
$$

is the degree distribution of a randomly chosen neighbor of nodes. Thus Eq. (4) gives the probability that a randomly chosen end of a randomly chosen link is infected. In the steady state, a nonvanishing solution for the prevalence is possible only for infection rates greater than (see $[12,13]$ )

$$
\lambda_{c}=\frac{\langle k\rangle}{\left\langle k^{2}\right\rangle} .
$$

For infection rates above the threshold, $\lambda>\lambda_{c}$, a finite fraction of the nodes is infected while for $\lambda \leq \lambda_{c}$ the disease dies out. ${ }^{1}$

For Erdős-Rényi (ER) networks, which obey a Poisson degree distribution [20], the threshold can be rewritten in the form $\lambda_{c}=1 /(\langle k\rangle+1)$. Moreover, for SF networks with $2<\gamma$ $\leq 3$ the second moment diverges and as a result the threshold vanishes. As a consequence such a system will always reach an endemic steady state for any nonzero infection rate $\lambda$ $>0$.

\section{B. Networks under rewiring dynamics}

During rewiring dynamics of a network the number of nodes and the number of links are unchanged but the links are stochastically detached from one node and reattached to another. In our model the process of rewiring a randomly chosen end of a link from a node with degree $k$ to a node with degree $k^{\prime}$ occurs with rate $u(k) v\left(k^{\prime}\right)$, where $u(k)$ is the detachment rate and $v\left(k^{\prime}\right)$ is the attachment rate. A schematic representation of the process is given in Fig. 1. These rates determine the steady state degree distribution of the network through the relation

\footnotetext{
${ }^{1}$ In the thermodynamic limit this is a transition to an absorbing state, but for finite size systems the only true steady state is one with zero prevalence [14]. As a result, in finite networks there is no true threshold but a crossover infection rate which can be calculated for a quasistationary state.
} 


$$
\mathcal{P}(k)=\frac{\prod_{k^{\prime}=0}^{k-1} v\left(k^{\prime}\right)}{\langle v\rangle^{k}} \frac{\langle u\rangle^{k}}{\prod_{k^{\prime}=1}^{k} u\left(k^{\prime}\right)} \mathcal{P}(0),
$$

where $\mathcal{P}(0)$ is the concentration of isolated nodes, as determined from the normalization condition of the distribution function. The above result [Eq. (7)] can be derived from the master equation for the node degree distribution [16].

Under such rewiring dynamics, the resulting networks are uncorrelated in the sense that the joint probability $\pi\left(k, k^{\prime}\right)$ that the ends of a randomly chosen link are nodes of degree $k$ and $k^{\prime}$ factorizes to

$$
\pi\left(k, k^{\prime}\right)=\mathcal{P}^{*}(k) \mathcal{P}^{*}\left(k^{\prime}\right)
$$

where $\mathcal{P}^{*}(k)$ is defined in Eq. (5).

By choosing the proper attachment and detachment rates one can create an evolving network with a constant size and any desired degree distribution. One such choice of rewiring yields an evolving ER type network. This is achieved by choosing a link at random and rewiring one of it's randomly chosen ends to a randomly chosen node. This rewiring scheme has a constant attachment rate and a linearly preferential detachment rate. One can easily verify by using Eq. (7) that the choice

$$
\begin{gathered}
v(k)=\frac{1}{N}, \\
u(k)=k
\end{gathered}
$$

indeed yields a Poisson degree distribution.

Through the use of such rewiring dynamics we can create an uncorrelated SF network with any desired exponent in the power-law distribution. In what follows we work with rewiring dynamics similar to that of zero-range processes (ZRP) $[17,18]$, where the rewiring rate does not depend on the destination site, i.e., $v(k)=1 / N$. As a further specification we consider detachment rates of the form

$$
u(k)=1+\frac{b}{k}
$$

with $b$ as a parameter of the dynamics. In this case, for a specific choice of the average number of links [given by $\langle k\rangle=1 /(b-2)]$ the underlying zero-range process exhibits critical behavior in which the steady state degree distribution is a power law $\mathcal{P}(k) \sim k^{-b}$ at large $k$. At lower average link numbers the steady state distribution decays exponentially with $k$ while at larger averages a hub becomes present which is linked to a finite fraction of the nodes in the network $[17,18]$.

In order to be able to control the critical value of $\langle k\rangle$ for a given value of $b$ one can make a slight modification in the dynamics by considering

$$
u(k)=\left\{\begin{array}{ll}
1+\frac{b}{k_{0}} & 0<k \leq k_{0} \\
1+\frac{b}{k} & k>k_{0}
\end{array} .\right.
$$

Due to the same asymptotic behavior of Eqs. (10) and (11) this modification does not change the power-law tail of the stationary degree distribution. In this case the critical value of $\langle k\rangle$ can be obtained numerically [17]. Note that for rewiring rates of the form Eqs. (10) and (11), $\langle u\rangle=1$ at criticality [17].

It is important to note that the dynamics, as defined, allows for multiple link between two nodes (melons) and links that connect a node with itself (tadpoles). By not allowing for melons and tadpoles we are introducing an effective preferential attachment rate $v(k)=1-k / N$ as opposed to a constant rate as given in Eq. (9). This rate takes into account the fact that the neighbors of a node of degree $k$ are not available as target nodes for the rewired link. The preferential attachment rate means that a highly connected node has a lower rate of attachment than a node with a lower connectivity and induces disassortative, or negative correlations. It can be shown using Eq. (7) that this attachment rate imposes a Gaussian cutoff on the degree distribution of the form

$$
\mathcal{P}^{\prime}(k)=e^{-k^{2} / 2 N} \mathcal{P}(k),
$$

where $\mathcal{P}(k)$ is the degree distribution for similar dynamics which allows for tadpoles and melons. For ER type networks and for SF networks with $\gamma>3$ the fraction of melons and tadpoles vanishes in the thermodynamic limit [21]. However, for SF networks with $\gamma \leq 3$ the number of melons and tadpoles diverges and cannot be neglected. In this work we use $v(k)=1 / N$, for which tad poles and melons are allowed.

\section{EPIDEMIC SPREADING ON EVOLVING NETWORKS}

Our aim is to consider a model of epidemic spreading on a network which is changing in time. As a consequence, a given node is no longer connected to a static set of neighbors but to a dynamic one, and the degree $k$ of the node also fluctuates. Previous work on epidemic spreading on evolving networks [22-25] concentrated mainly on rewiring dynamics resulting from the adaptation of the network to the disease. In these models the rewiring dynamics depend on the state of the nodes, i.e., the infection process. However, recent work [26] suggests that rewiring dynamics that are independent of the state of the nodes can suppress the spread of disease in the network. Here we consider such a model where the rewiring dynamics is independent of the infection process. To be more specific, we consider a ZRP-like rewiring dynamics for the network with rates of the form Eq. (11) and $v(k)$ $=1 / N$. For a specific value of $\langle k\rangle$ this results in a power-law degree distribution for the steady state of the network (which depends on $k_{0}$ ) with $\mathcal{P}(k) \sim k^{-b}$ for large $k$. In addition, we introduce a parameter $\nu$, which describes the overall time scale of the rewiring process as compared to that of the infection process. The rewiring rate from a node of degree $k$ 
then becomes $\nu u(k)$. For $\nu=0$ the model reduces to epidemic spreading on a static network, whereas for $\nu \rightarrow \infty$, due to the fast mixing we expect a mean-field-like behavior for the infection process, where neighbors change very rapidly. We note here that we always consider the case where the network is in a stationary state with respect to the rewiring dynamics, which requires a diverging equilibration time for $\nu \rightarrow 0$.

\section{A. Mean-field results}

In order to account for the rewiring dynamics, Eq. (3) has to be modified as follows:

$$
\begin{aligned}
\frac{\partial \rho_{k}}{\partial t}= & -\rho_{k}+\lambda k \ell\left(1-\rho_{k}\right)-\nu \rho_{k}\left(u_{k}+\langle u\rangle\right) \\
& +\nu\left[\rho_{k+1} \frac{\mathcal{P}(k+1)}{\mathcal{P}(k)} u_{k+1}+\rho_{k-1} \frac{\mathcal{P}(k-1)}{\mathcal{P}(k)}\langle u\rangle\right],
\end{aligned}
$$

where we used the fact that the attachment rate is equal to the average detachment rate $\langle u\rangle$. By multiplying Eq. (13) with $\mathcal{P}(k)$ and summing up for all $k$ one obtains

$$
\rho=\lambda\langle k\rangle \ell(1-\ell)
$$

in the stationary state. For infinitesimal $\rho$ and $\ell$ (at the threshold) this reduces to

$$
\rho=\lambda_{c}\langle k\rangle \ell .
$$

Note that one can rewrite definition (4) of $\ell$ as

$$
\ell \equiv \rho\langle k\rangle_{\text {inf }} /\langle k\rangle,
$$

where $\langle\cdot\rangle_{\text {inf }}$ denotes an average in the ensemble of infected nodes. We define the average of a quantity $x$ in the ensemble of infected nodes as

$$
\langle x\rangle_{\text {inf }}=\rho^{-1} \sum_{k} x \rho_{k} \mathcal{P}(k) .
$$

Using this, Eq. (15) takes the following simple form:

$$
\lambda_{c}^{-1}=\langle k\rangle_{\text {inf }} .
$$

On the other hand, multiplying Eq. (13) by $k \mathcal{P}(k)$ and summing up over all $k$ one obtains the following equation for the steady state near the threshold (the second order term is neglected):

$$
0=-\rho\langle k\rangle_{\mathrm{inf}}+\lambda_{c}\left\langle k^{2}\right\rangle \ell+\nu \rho\left(\langle u\rangle-\langle u\rangle_{\mathrm{inf}}\right) .
$$

By using Eq. (18) for the first and Eq. (15) for the second term, the above equation reduces to

$$
\lambda_{c}^{-1}=\frac{\left\langle k^{2}\right\rangle}{\langle k\rangle}+\nu\left(\langle u\rangle-\langle u\rangle_{\text {inf }}\right) .
$$

Whether or not there exists a nonzero infection rate threshold can be easily deduced from this equation. For the rewiring rates [Eq. (11)] both $\langle u\rangle$ and $\langle u\rangle_{\text {inf }}$ are finite. Thus there exists a finite positive threshold as long as $\left\langle k^{2}\right\rangle$ is finite, namely, for networks with $\gamma>3$. In this case the rewiring rate $\nu$ affects the threshold quite strongly. On the other hand for networks with $2<\gamma \leq 3,\left\langle k^{2}\right\rangle$ diverges and $\lambda_{c}$ vanishes.

It is obvious that for $\nu \rightarrow 0$, Eq. (20) reduces to $\lambda_{c}$ $=\langle k\rangle /\left\langle k^{2}\right\rangle$ as discussed before. In the other extreme case, when $\nu \rightarrow \infty$, due to the fast rewiring we expect that the degree distribution of infected and noninfected nodes become identical. This would imply that $\rho_{k}=\rho$ for all $k$ and $\langle k\rangle_{\text {inf }}$ $=\langle k\rangle$. Therefore, based on Eq. (18), one has $\lambda_{c}=\langle k\rangle^{-1}$. Note also that in this infinite rewiring limit the rhs of Eq. (20) is nontrivial, since $\nu \rightarrow \infty$, while $\langle u\rangle-\langle u\rangle_{\text {inf }}$ is expected to vanish.

It is important to note that whereas for small values of $\nu$ the MF approximation, that we use throughout this section, is not necessarily valid. However, in the $\nu \rightarrow \infty$ limit the fast rewiring ruins all the correlations in the system, and the MF approximation is expected to become asymptotically exact.

As shown by Eq. (18) the threshold is determined by the degree distribution of infected nodes at the transition point. In the following we attempt to get a deeper understanding of how this distribution changes with the rewiring rate $\nu$. For this reason we define

$$
r_{k}=\lim _{\lambda \searrow \lambda_{c}} \frac{\rho_{k}}{\rho},
$$

and assume that this quantity is finite for all $k$. This implies the following normalization for $r_{k}$,

$$
\sum_{k=0}^{\infty} \mathcal{P}(k) r_{k}=1
$$

It is easy to see that

$$
\mathcal{P}(k) r_{k}=\tilde{\mathcal{P}}(k)
$$

is the degree distribution of infected nodes close to the threshold.

Equation (13) together with the steady state relation

$$
\frac{\mathcal{P}(k)}{\mathcal{P}(k+1)}=\frac{u_{k+1}}{\langle u\rangle}
$$

implies

$$
0=-\rho_{k}+\lambda k \ell\left(1-\rho_{k}\right)+\nu\left[\langle u\rangle \rho_{k+1}+u_{k} \rho_{k-1}-\left(\langle u\rangle+u_{k}\right) \rho_{k}\right]
$$

for the steady state. By dividing the above equation with $\rho$ and taking the limit $\lambda \searrow \lambda_{c}$ we obtain

$$
0=-r_{k}+\frac{k}{\langle k\rangle}+\nu\left[r_{k+1}\langle u\rangle+r_{k-1} u_{k}-r_{k}\left(u_{k}+\langle u\rangle\right)\right] .
$$

Here we used the definition (21) of $r_{k}$ and Eq. (15) for the second term of Eq. (25) (the second order term is neglected).

After solving the above set of equations for $r_{k}$ one can determine $\lambda_{c}$ from Eqs. (18) and (21) as

$$
\lambda_{c}^{-1}=\sum_{k} k r_{k} \mathcal{P}(k) \text {. }
$$

One can immediately see that in the $\nu \rightarrow \infty$ limit the solution of Eq. (26) is $r_{k}=1$. This implies $\tilde{\mathcal{P}}=\mathcal{P}$ which results in the already noted limiting behavior with $\lambda_{c}=1 /\langle k\rangle$. On the other hand, in the case of a static network, where $\nu=0, \rho_{k}$ is 

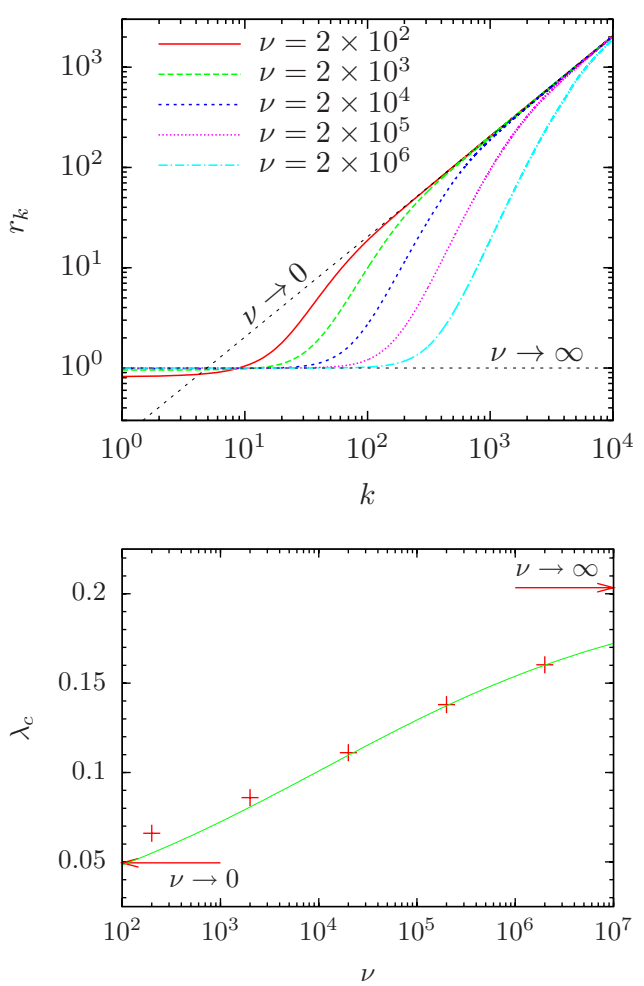

FIG. 2. (Color online) Top: $r_{k}$ is plotted against $k$ for various rewiring rates. The curves represent increasing rewiring rates from left to right. The set of Eqs. (26) is solved numerically with $b$ $=3.5$ and $k_{0}=15$ for the critical case, which corresponds to $\langle u\rangle=1$, $\langle k\rangle=4.917454$, and $\left\langle k^{2}\right\rangle=99.39410$. Bottom: The threshold $\lambda_{c}$ as a function of $\nu$. It was calculated numerically using Eq. (27) with the same parameters. The analytically derived $\nu \gg 1$ limiting formula (33) is also plotted (full line) with $C=50$, which gives a good fit.

proportional to $k$ near the threshold, implying $r_{k}=k /\langle k\rangle$ and $\widetilde{\mathcal{P}}=\mathcal{P}^{*}$, which results in $\lambda_{c}=\langle k\rangle /\left\langle k^{2}\right\rangle$.

Numerical solutions of Eq. (26) for intermediate values of $\nu$ are shown in Fig. 2. One can see that for a large but finite $\nu$ there are two crossover values of $k$. For $k$ below some value $k_{1}$ one has $r_{k}=1$ and $\widetilde{\mathcal{P}}=\mathcal{P}$ (infinite rewiring), whereas for $k$ larger than some other value $k_{2}$, one has $r_{k}=k /\langle k\rangle$ and $\widetilde{\mathcal{P}}=\mathcal{P}^{*}$ (static). Between $k_{1}$ and $k_{2}$ we find an intermediate regime, which connects the two extreme cases. The crossover values $k_{1}$ and $k_{2}$ increase with increasing $\nu$.

Using these numerical solutions for $r_{k}$ we calculated numerically the effect of rewiring, in networks with $\gamma>3$, on the threshold $\lambda_{c}$ by using Eq. (27). Results are shown in Fig. 2. One can clearly see that as the rewiring rate increases the threshold increases.

A more careful analysis of the set of Eqs. (26) for $\nu \gg 1$ shows that there are two important limiting cases:

(i) For large $\nu$ the third term balances the first one, to leading order, whereas the second term is small compared to them. In this limiting case

$$
r_{k}=1+\frac{k^{3}}{3 \nu\langle k\rangle(b-2)} .
$$

This is valid, when $\nu \gg k^{2}$.

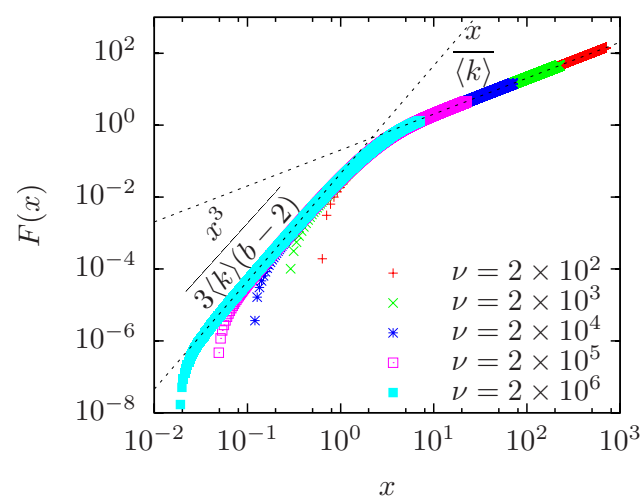

FIG. 3. (Color online) $F(x)=\left(r_{k}-1\right) \nu^{-1 / 2}$ is plotted against $k \nu^{-1 / 2}$ using the data of Fig. 2. The data collapse confirms the scaling form. The analytically obtained limiting forms [Eq. (31)] are plotted with dashed lines.

(ii) For large $k$ the second term balances the first one, to leading order, and the third term is small. In this case

$$
r_{k}=\frac{k}{\langle k\rangle}-\frac{\nu b}{\langle k\rangle k} \text {. }
$$

This is valid, when $\nu \ll k^{2}$.

The above forms suggest, that for $\nu \gg 1$ the solution has a scaling form

$$
r_{k}=1+\nu^{1 / 2} F\left(k \nu^{-1 / 2}\right)
$$

This is verified by the data collapse shown in Fig. 3, where $\left(r_{k}-1\right) \nu^{-1 / 2}$ is plotted as a function of $k \nu^{-1 / 2}$. From Eqs. (28) and (29) we obtain that

$$
F(x)= \begin{cases}\frac{x^{3}}{3\langle k\rangle(b-2)} & x \ll 1 \\ \frac{x}{\langle k\rangle} & x \gg 1 .\end{cases}
$$

This limiting behavior is apparent in Fig. 3 .

In order to obtain the functional form of $F(x)$ in the intermediate regime $[x=\mathcal{O}(1)]$ one can take the continuous scaling limit of Eq. (26) with $k \rightarrow \infty, \nu \rightarrow \infty$, and $k / \sqrt{\nu}=x$ $=$ const. This results in the following ordinary differential equation.

$$
F^{\prime \prime}(x)-\frac{b}{x} F^{\prime}(x)-F(x)+\frac{x}{\langle k\rangle}=0 .
$$

Using the scaling form [Eq. (30)] one can easily show that in the $\nu \gg 1$ limit

$$
\lambda_{c}^{-1}=\langle k\rangle_{\mathrm{inf}}=\sum_{k} k r_{k} \mathcal{P}(k)=\langle k\rangle+C \nu^{3-b / 2},
$$

where $C$ is some constant (this is valid only when $\lambda_{c}^{-1}$ is finite, i.e., for $b>3$ ). The numerically calculated $\lambda_{c}$ fits on this form with $C \approx 50$ (see Fig. 2).

\section{B. Simulations}

In this section we use numerical simulations of finite SF networks to investigate the effect of rewiring on prevalence. 

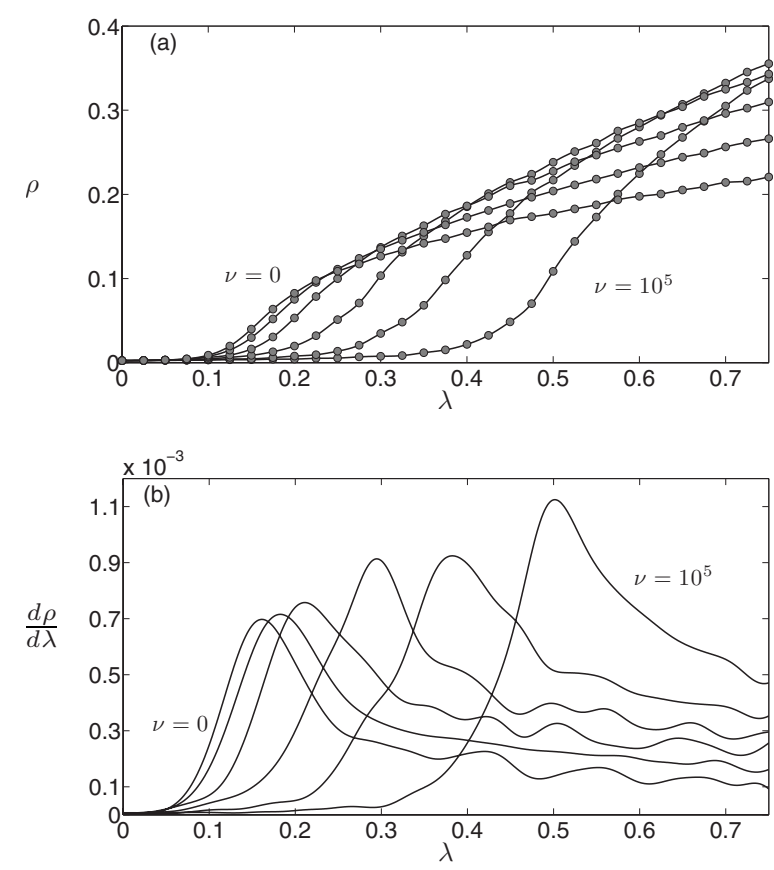

FIG. 4. The prevalence $\rho$ (a) and the derivative of the prevalence with respect to the infection rate $\mathrm{d} \rho / \mathrm{d} \lambda$ (b) are plotted as a function of the infection rate for a network of size $N=200,\langle k\rangle=2$ and rewiring dynamics [Eq. (11)] with $b=2.5$ and $k_{0}=1$ for which $\left\langle k^{2}\right\rangle \approx 58$. Curves for rewiring rates $\nu=0,10,10^{2}, 10^{3}, 10^{4}, 10^{5}$ are represented by full lines from left to right respectively. For clarity, the derivative was calculated using smoothed interpolated data of the prevalence.

In order to compare the simulation results to the MF predictions described in Sec. III A we simulate two types of SF networks; networks with $\gamma>3$, for which the MF predicts a threshold $\lambda_{c}$ in the infection rate, and networks with $2<\gamma$ $\leq 3$, for which the MF predicts no threshold. We use these two types even though it was recently shown [11] that in the thermodynamic limit no threshold exists for any SF network, i.e., for any $\gamma$.

The networks were constructed using two sets of parameter values for the rewiring dynamics [Eq. (11)]. As an example of a network with $2<\gamma \leq 3$ we use the dynamics with $v(k)=1 / N, u(k)=1+b / k\left(k_{0}=1\right)$ and $b=2.5$. As described previously, $\langle k\rangle=1 /(b-2)=2$ corresponds to the critical average value of the underlying zero-range process for which the steady state degree distribution is a power law with $\gamma=2.5$. The resulting prevalence as a function of the infection rate is plotted in Fig. 4(a) for various rewiring rates.

As an example of a network with $\gamma>3$ we used the dynamics [Eq. (11)] with $\langle k\rangle \approx 4.917, b=3.5$ and $k_{0}=15$, corresponding to the critical average value of the underlying zerorange process for which the steady state degree distribution is a power law with $\gamma=3.5$. The resulting prevalence as a function of the infection rate is plotted in Fig. 5(a) for various rewiring rates.

For a network of finite size, there is no true threshold but a crossover infection rate which is obtained for a quasistationary state. In simulations one can identify this crossover value $\lambda_{c}(N, L)$ as the point where the (numerically obtained) derivative $d \rho / d \lambda$ takes its maximum. With such a definition
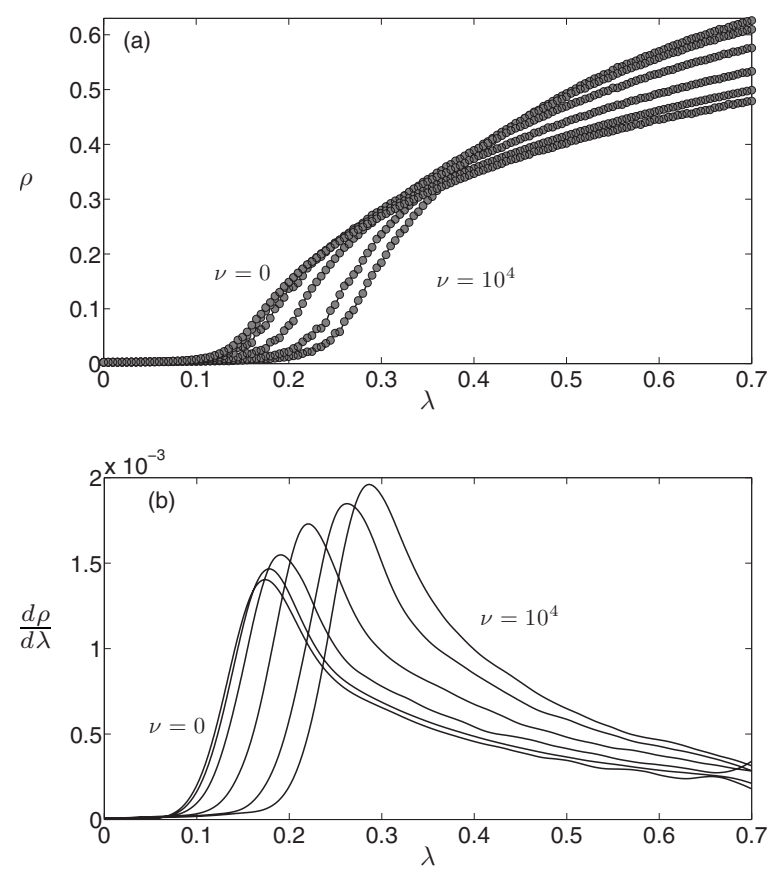

FIG. 5. The prevalence $\rho$ (a) and the derivative of the prevalence with respect to the infection rate $d \rho / d \lambda$ (b) are plotted as a function of the infection rate for a network of size $N=200,\langle k\rangle$ $=4.9$ and rewiring dynamics [Eq. (11)] with $b=3.5$ and $k_{0}=15$ for which $\left\langle k^{2}\right\rangle \approx 73$. Curves for rewiring rates $\nu=0,10,10^{2}, 10^{3}, 10^{4}$ are represented by full lines from left to right, respectively. For clarity, the derivative was calculated using smoothed interpolated data of the prevalence.

$\lambda_{c}(N, L) \rightarrow \lambda_{c}$ in the thermodynamic limit. One can see in Fig. 4(b), corresponding to $2<\gamma<3$, that as the rewiring rate increases $\lambda_{c}(N, L)$ increases from approximately $\langle k\rangle /\left\langle k^{2}\right\rangle \approx 0.04$ toward $1 /\langle k\rangle=0.5$. Similarly, one can see in Fig. 5(b), corresponding to $\gamma>3$, that as the rewiring rate increases $\lambda_{c}(N, L)$ increases from approximately $\langle k\rangle /\left\langle k^{2}\right\rangle$ $\approx 0.07$ toward $1 /\langle k\rangle=0.2$. Even though these simulation results do not prove our result that $\lambda_{c}(N, L) \rightarrow 1 /\langle k\rangle$ as $\nu$ increases they seem to suggest that this is the case.

As described before, rewiring has an effect on the threshold that depends on the network degree distribution. For degree distributions with a finite second moment the threshold $\lambda_{c}$ is affected even for rewiring rates of order 1. For networks with a diverging second moment (in the thermodynamic limit) the threshold is affected only for $\nu \rightarrow \infty$. Most real world networks, however, are finite for which the second moment scales as $\left\langle k^{2}\right\rangle \sim N^{(3-\gamma) /(\gamma-1)}$. What this means is that a finite rewiring rate has an observable effect on the crossover rate $\lambda_{c}(N, L)$.

In the simulations a very weak external source of infection was introduced in order to prevent the system from fluctuating into the absorbing state. There are several other methods of simulating an absorbing phase transition and computing from it the value of the threshold which are reviewed in [27].

Note that for the considered value of $b=2.5,\left\langle k^{2}\right\rangle$ diverges in the thermodynamic limit, therefore, based on the MF results, the threshold is expected to vanish for $\nu=0$. However, for a finite system one expects a finite crossover value for $\lambda$, 


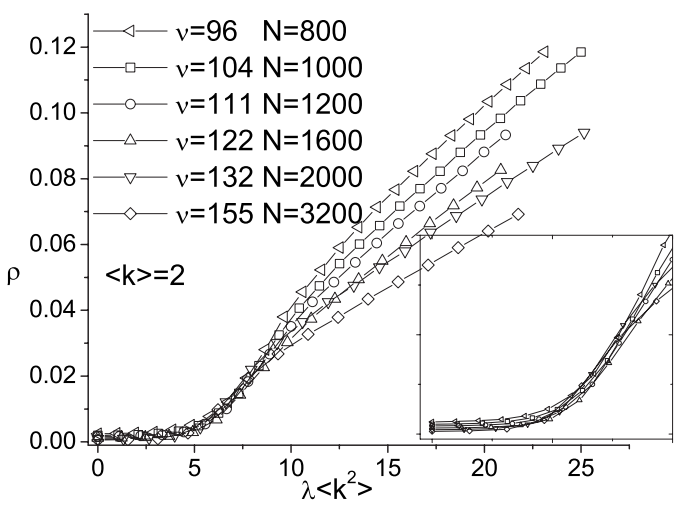

FIG. 6. The prevalence as a function of the scaled infection rate $\lambda_{c}\left\langle k^{2}\right\rangle$ for a network of different sizes $N,\langle k\rangle=2$ and rewiring dynamics [Eq. (11)] with $b=2.5$ and $k_{0}=1$ plotted for different rewiring rates where for each network of size $N$ the rewiring rate is $\nu$ $=\left\langle k^{2}\right\rangle$. The behavior near the threshold is replotted on a finer scale and is given in the inset.

which is of order $1 /\left\langle k^{2}\right\rangle$. On the other hand, for $\nu \gg\left\langle k^{2}\right\rangle$ the crossover value should increase up to $1 /\langle k\rangle$. It is interesting to examine how the crossover $\lambda$ changes if the rewiring rate is of order $\left\langle k^{2}\right\rangle$. To this end we performed simulations with $\nu \sim\left\langle k^{2}\right\rangle$. We found that the threshold scales as $\lambda_{c}^{-1} \sim\left\langle k^{2}\right\rangle$. The corresponding data collapse is presented in Fig. 6 where the prevalence is plotted as a function of a scaled infection rate $\lambda_{c}\left\langle k^{2}\right\rangle$ for networks of different sizes with a rewiring rate equal to the second moment of the degree distribution $\nu$ $=\left\langle k^{2}\right\rangle$.

In Figs. 7 and 8 the simulation results are compared to the numerical solution of Eq. (14) for $b=2.5$ and $b=3.5$ for both a static network $(\nu=0)$ and for $\nu=10^{4}$. The numerical calculation of the MF contact equation was carried out by solving Eq. (13) for each infection rate. The degree distribution used in the calculation was taken from the simulation results. For both the $b=2.5$ and $b=3.5$ cases the MF solution for $\nu$ $=10^{4}$ agrees quite well with the simulation results which supports our general argument that as the rewiring rate increases, compared to the infection process, both the degree of a node and fluctuations average out such that the MF approximation better describes the process.

\section{CONCLUSIONS}

The effect of network dynamics on epidemic spreading has been studied using mean-field analysis and numerical

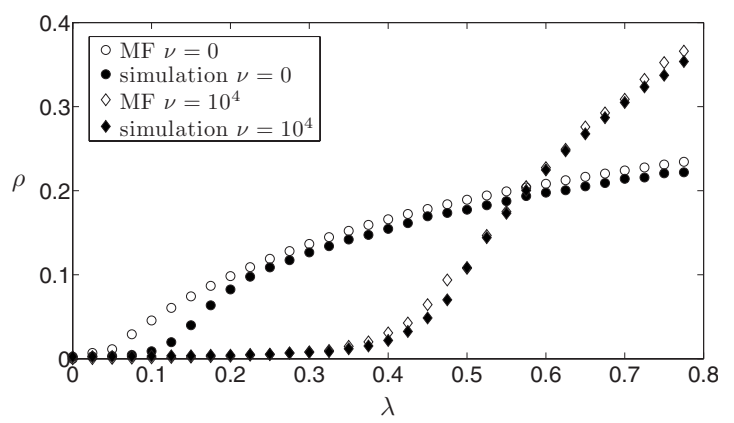

FIG. 7. The prevalence as a function of the infection rate for a network of size $N=200,\langle k\rangle=2$ and rewiring dynamics [Eq. (11)] with $b=2.5$ and $k_{0}=1$. The simulation results for $\nu=0$ and $\nu=10^{4}$ are compared with the numerical solution of Eq. (14).

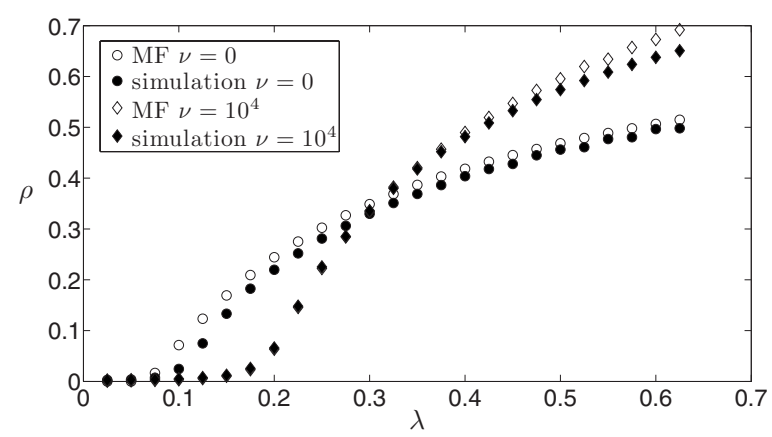

FIG. 8. The prevalence as a function of the infection rate for a network of size $N=200,\langle k\rangle \approx 4.917$ and rewiring dynamics [Eq. (11)] with $b=3.5$ and $k_{0}=15$. The simulation results for $\nu=0$ and $\nu=10^{4}$ are compared with the numerical solution of Eq. (14).

simulations. In particular we considered epidemic spreading over SF networks with rewiring dynamics.

We have shown that the introduction of rewiring affects the threshold for an endemic state of a network. This is a surprising result that an evolving network is fitter with respect to disease the faster it is rewired. This result is general to any network with a general degree distribution.

One can understand this counter intuitive result by associating the second moment of the degree distribution with the heterogeneity of a network. The more heterogeneous is a network the larger is the fraction of highly connected nodes which mediate the infection process. The introduction of rewiring effectively averages out the heterogeneity and creates an effective homogeneous network, with respect to the infection process, where each node has an effective average degree $k=\langle k\rangle$.

Different networks differ in the rate of rewiring that is required for a change of the threshold. We have shown, under the MF approximation, that for networks with different degree distributions the relevant quantity is the second moment of the degree distribution. Only if the rewiring is larger than the second moment $\nu \gtrsim\left\langle k^{2}\right\rangle$ then the threshold, as resulting form the MF calculation, is affected and is increased from $\lambda_{c}^{-1}=\langle k\rangle /\left\langle k^{2}\right\rangle$ to $\lambda_{c}^{-1}=1 /\langle k\rangle$. For homogeneous networks, such as ER networks, rewiring has little effect on the behavior of the disease since $\langle k\rangle^{2} \approx\left\langle k^{2}\right\rangle$. For heterogeneous networks, for which $\left\langle k^{2}\right\rangle \gg\langle k\rangle$, rewiring has a larger effect. For example, we have argued that in the thermodynamic limit the threshold for SF networks with $\gamma>3$ will increase continuously with the rewiring rate. On the other hand, for SF networks with $\gamma<3$ there is no threshold in the thermodynamic limit, except for an infinite rewiring rate.

Our analysis of the effect of rewiring has been carried out under a MF approximation, where the effects of the third and higher moments of the degree distribution are neglected. While this approximation is valid for well behaved degree distributions, it fails for SF networks. Recent research [11] has shown, through direct calculation, that for SF networks no threshold exists even for distributions with $\gamma>3$. What this suggests is that, in the thermodynamic limit, for any SF network only an infinite rewiring rate would effect the threshold. 
Nevertheless, most real life networks are finite, for which we have shown that even though a true threshold does not exist, the crossover infection rate $\lambda_{c}(N, L)$ increases as we increase the rewiring rate. Moreover, there are many heterogeneous networks which are not SF for which a threshold exists. Therefore, we can conclude that for most real life networks a finite rewiring rate will increase the resistance of the network to epidemic spreading.

\section{ACKNOWLEDGMENTS}

The support of the Israel Science Foundation (ISF) is gratefully acknowledged. We thank Oren Shriki for discussions. Y.S. thanks Aaron Clauset for computing resources. A. Rákos acknowledges financial support from the Hungarian Scientific Research Fund (OTKA) Grants No. PD-72604 and No. PD-78433, and from the Hungarian Academy of Sciences.
[1] F. Liljeros, C. R. Edling, L. A. N. Amaral, H. E. Stanley, and Y. Aberg, Nature (London) 411, 907 (2001).

[2] A. L. Lloyd and R. M. May, Science 292, 1316 (2001).

[3] M. E. J. Newman, S. Forrest, and J. Balthrop, Phys. Rev. E 66, 035101 (2002).

[4] S. N. Dorogovtsev, A. V. Goltsev, and J. F. F. Mendes, Rev. Mod. Phys. 80, 1275 (2008).

[5] A. Barrat, M. Barthelemy, and A. Vespignani, Dynamical Processes in Complex Networks (Cambridge University Press, Cambridge, England, 2008).

[6] A.-L. Barabási and R. Albert, Science 286, 509 (1999).

[7] A.-L. Barabási, R. Albert, and H. Jeong, Physica A 272, 173 (1999).

[8] B. A. Huberman and L. A. Adamic, Nature (London) 401, 131 (1999).

[9] A.-L. Barabási, R. Albert, and H. Jeong, Physica A 281, 69 (2000).

[10] R. Albert and A.-L. Barabási, Rev. Mod. Phys. 74, 47 (2002).

[11] S. Chatterjee and R. Durrett, Ann. Probab. 37, 2332 (2009).

[12] R. Pastor-Satorras and A. Vespignani, Phys. Rev. Lett. 86, 3200 (2001).

[13] R. Pastor-Satorras and A. Vespignani, Phys. Rev. E 63, 066117 (2001).

[14] R. Pastor-Satorras and A. Vespignani, Phys. Rev. E 65, 035108 (2002).

[15] M. Boguñá, R. Pastor-Satorras, and A. Vespignani, Lect. Notes
Phys. 625 (2003)

[16] S. N. Dorogovtsev and J. F. F. Mendes, Evolution of Networks (Oxford University Press, New York, 2003).

[17] M. R. Evans and T. Hanney, J. Phys. A 38, R195 (2005).

[18] A. G. Angel, M. R. Evans, E. Levine, and D. Mukamel, Phys. Rev. E 72, 046132 (2005).

[19] R. Pastor-Satorras and A. Vespignani, Phys. Rev. E 65, 036104 (2002).

[20] P. Erdős and A. Rényi, Publ. Math., Inst. Hautes Etud. Sci. 5, 17 (1960).

[21] M. Boguñá, R. Pastor-Satorras, and A. Vespignani, Eur. Phys. J. B 38, 205 (2004).

[22] T. Gross, C. J. D. D'Lima, and B. Blasius, Phys. Rev. Lett. 96, 208701 (2006).

[23] E. Volz and L. A. Meyers, Proc. R. Soc. London, Ser. B 274, 2925 (2007).

[24] L. B. Shaw and I. B. Schwartz, Phys. Rev. E 77, 066101 (2008).

[25] L. B. Shaw and I. B. Schwartz, in Adaptive Networks: Theory, Models and Applications, Studies on Complexity Series, edited by T. Gross and H. Sayama (Springer/NECSI, Heidelber, 2009).

[26] N. H. Fefferman and K. L. Ng, Phys. Rev. E 76, 031919 (2007).

[27] M. M. de Oliveira and R. Dickman, Phys. Rev. E 71, 016129 (2005). 